\title{
Metachronous gastric cancers after endoscopic resection: how effective is annual endoscopic surveillance?
}

\author{
Takeshi Nakajima, Ichiro Oda, Takuji Gotoda, Hisanao Hamanaka, Takako Eguchi, Chizu Yokoi, \\ and DAIZO SAITO \\ Endoscopy Division, National Cancer Center Hospital, 5-1-1 Tsukiji, Chuo-ku, Tokyo 104-0045, Japan
}

\begin{abstract}
Background. Endoscopic resection (ER) has been widely accepted in Japan as a less invasive treatment for early gastric cancer, but the incidence of subsequent metachronous gastric cancer (MGC) and the appropriate endoscopic follow-up interval after ER have not been determined as yet. In this study, we investigated the incidence of MGC after ER and assessed our annual endoscopic surveillance program after ER.

Methods. We studied the clinicopathological features of 633 consecutive ER patients (575 with a single lesion and 58 with synchronous multiple lesions) treated at our institution from 1987 through 2002, after excluding 158 patients who underwent additional surgery due to noncurative ERs, 180 patients whose surveillance periods were less than 1 year, 1 patient with hereditary non-polyposis colorectal cancer, and 1 patient with gastric tube cancer. We defined a second cancer found within 1 year after ER as "synchronous" and a second cancer found after 1 year as "metachronous."

Results. First MGCs had an overall incidence of $8.2 \%$ (52 out of 633 patients); the annual incidence was constant, and the cumulative 3 -year incidence was $5.9 \%$. The average time to the discovery of a first MGC after the initial ER was $3.1 \pm 1.7$ years (range, 1-8.6 years). Almost all first MGCs (96.2\%, 50 out of 52 lesions) were treated curatively with repeat ER.

Conclusion. In order to detect MGC at a stage early enough for a curative repeat $\mathbf{E R}$, an annual endoscopic surveillance program is both practical and effective for post-ER patients.
\end{abstract}

Key words Early gastric cancer - Endoscopic mucosal resection $\cdot$ Metachronous cancer

\section{Introduction}

Recent advances in endoscopic examinations have made it possible to detect early gastric cancer (EGC). Due to the resultant increase in the number of EGCs,

Offprint requests to: D. Saito

Received: August 19, 2005 / Accepted: February 24, 2006 endoscopic resection (ER), which includes endoscopic mucosal resection (EMR) and endoscopic submucosal dissection (ESD), is now widely accepted in Japan as a less invasive treatment for EGCs, without the risk of regional lymph-node metastasis [1-3].

In Studies conducted on patients with surgically resected stomachs, the synchronous multiplicity of gastric cancer is well known, and its frequency has been reported to range from $4.8 \%$ to $20.9 \%$ at several institutions [4-10]. In addition, the incidence of new metachronous gastric cancer (MGC) in the remnant stomach after surgery for gastric cancer ranges from $1.8 \%$ to $2.4 \%[11,12]$. Consequently, the remnant stomach is at high risk for the development of MGC.

Although ER contributes to preserving most of the stomach, the risk of MGC developing at other sites in the stomach in the post-ER patient has become a major problem. There have been only a few reports regarding the incidence of MGC after ER. At present, we perform annual endoscopic surveillance for post-ER patients, but the proper interval of examination after ER is still not known.

\section{Patients and methods}

\section{Patients}

The aim of this study was to investigate the clinicopathological features and incidence of MGC after ER and to assess our annual endoscopic surveillance program following ER. A total of 1129 EGCs in 973 patients were treated by ER from 1987 through 2002 at our institution. Of these patients, 633 (575 with a single lesion and 58 with synchronous multiple lesions) were evaluated in this study. We excluded 158 patients who underwent additional surgery due to noncurative ERs, 180 patients whose surveillance periods were less than 1 year, 1 patient with hereditary nonpolyposis colorectal 
Table 1. Indication criteria for endoscopic resection

Early gastric cancer meeting all of the following:

(1) Differentiated adenocarcinoma (biopsy)

(2) No apparent invasive findings (endoscopy)

(3) Less than $3 \mathrm{~cm}$ in size regardless of ulcer findings (endoscopy) or without ulcer findings regardless of tumor size (endoscopy)

Table 2. Expanded histological criteria for endoscopic resection

(1) Differentiated adenocarcinoma

(2) No lymphatic or venous invasion

(3) Intramucosal cancer regardless of tumor size without ulcer finding or intramucosal cancer $\leq 30 \mathrm{~mm}$ in size with ulcer finding or minute submucosal cancer (sm1) $\leq 30 \mathrm{~mm}$ in size

cancer (HNPCC), and 1 patient with gastric tube cancer. We reviewed the clinical records, including endoscopic and histological reports, for all these patients. The average follow-up period after ER for the 633 study patients was $4.4 \pm 2.8$ years (range, $1.0-13.9$ years), the average age of the subjects was $66.5 \pm 9.0$ years (range, 35-93 years) and the male-to-female ratio was 4:1 (510 men and 123 women).

\section{Methods}

\section{ER and its evaluation}

At the beginning of this series of consecutive ERs, most of ERs were performed by the so-called "strip biopsy method," a relatively simple technique described previously [13]. Since 1997, however, a new ER procedure using an insulation-tipped diathermic knife [14] has been used in most patients at our institution. In this study, we evaluated patients with EGC consistent with the pre-ER indications shown in Table 1 [14-16]. "Curative resections" were evaluated using the expanded histological criteria shown in Table $2[15,16]$. The location of the tumors was defined as being in the upper, middle, or lower third of the stomach, based on the Japanese classification of gastric carcinoma [17]. The macroscopic types of EGCs were classified according to the Japanese Society for Gastroenterology Endoscopic Criteria, as type 0-I (protruded) or type 0-IIa (superficial elevated), which were both grouped together in our study as "elevated"; or as type 0-IIb (flat), type 0-IIc (superficial depressed), or type 0-IIa+IIc (elevated with central depression), which were all grouped together as "depressed" [18,19].

In view of the high risk of metastasis to lymph nodes or at distant sites, surgical intervention is strongly recommended for lesions with deep submucosal invasion, vessel involvement, or both. For patients who had undergone curative ER, we adopted an annual endoscopic surveillance program.

\section{Definition of multiple EGCs}

Although there is no consensus regarding the distinction between actual MGC and "missed" synchronous gastric cancer, we defined a second cancer found within 1 year after ER as "synchronous" and a second cancer found after more than 1 year as "metachronous." The diagnosis of multiple EGCs was defined according to the criteria of Moertel et al. [4].

\section{Statistics}

The follow-up period was measured from the date of the initial ER to the date of MGC diagnosis or the date of the last endoscopic examination. The factors affecting metachronous lesions were analyzed using the Kaplan-Meier method, and a log-rank test was conducted for age, sex, and multiple synchronous lesions found at the initial ER. A $P$ value of 0.05 was considered statistically significant.

\section{Aim of this study}

Focusing our attention on the incidence of metachronous lesions, we reviewed a large series of consecutive ERs performed at our institution. Based on data concerning the incidence, clinicopathological features, and outcomes of MGCs, we investigated the validity of our annual endoscopic surveillance program for postER patients.

\section{Results}

\section{Incidence}

The overall incidence for first MGCs was 8.2\% (52 out of 633 patients). The male-to-female patient ratio was approximately 8:1 (46 men and 6 women) and the average age at the time of the initial ER was $66.5 \pm 9.0$ years. The average interval between the discovery of MGC and the initial ER was $3.1 \pm 1.7$ years, with the longest interval after the initial ER being 8.6 years (Fig. 1). Fourteen of the 52 MGCs were followed up as adenomas or suspicion of carcinomas. Including and excluding these 14 lesions, the cumulative 3-year incidence of the first MGC was $5.9 \%$ and $4.0 \%$, respectively (Fig. 2). In terms of our statistical analysis of total MGCs, neither synchronous multiplicity of gastric cancer at the initial ER, sex, nor age significantly affected MGC incidence (Fig. 3A-C). In addition, 7 of the 52 patients with MGCs had double metachronous lesions and another 6 of them had a third metachronous lesion, all of which consisted of well- or moderately differenti- 


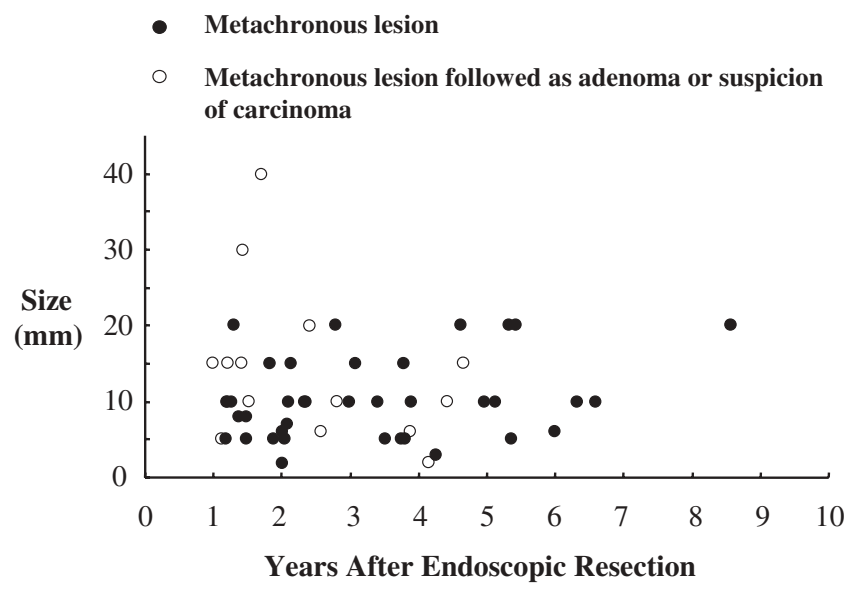

Fig. 1. Relationship between size of metachronous lesions and surveillance period. The longest interval after the initial endoscopic resection $(E R)$ was 8.6 years. Apart from two lesions, of $30 \mathrm{~mm}$ and $40 \mathrm{~mm}$, the average size of the metachronous gastric cancers (MGCs) was $10.3 \pm 5.4 \mathrm{~mm}$ (range, 2-20 mm). Closed circles, MGC; open circles, MGC followed as adenoma or suspicion of carcinoma

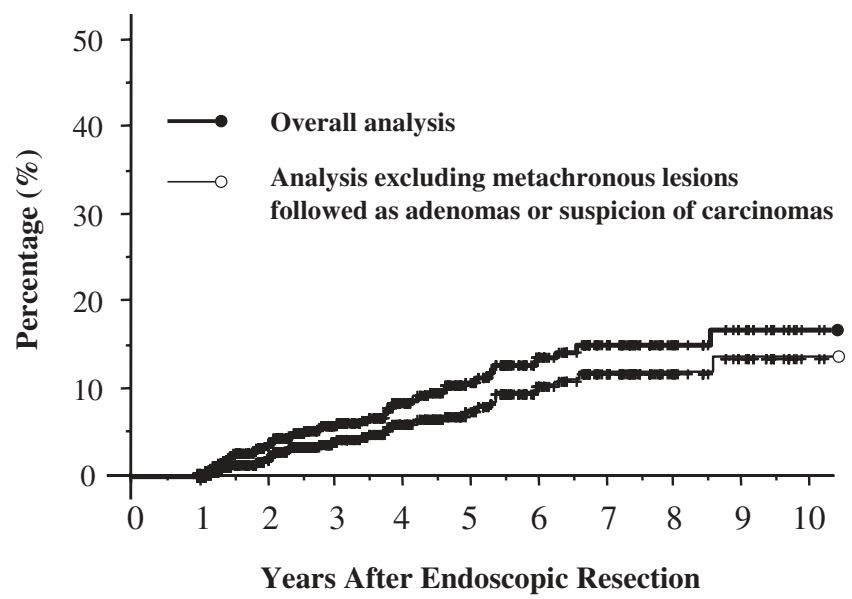

Fig. 2. Cumulative incidence of MGC. Including and excluding metachronous lesions followed as adenoma or suspicion of carcinoma, the cumulative 3-year incidence of the first MGC was $5.9 \%$ and $4.0 \%$, respectively, and the Kaplan-Meier curve was generally straight, suggesting that MGC incidence is constant

ated adenocarcinoma; all were resected curatively by repeat ER.

The first metachronous lesion occurred in the same location as the initial lesion in $40.4 \%(21 / 52)$ of the patients. In $63.5 \%(33 / 52)$ of the patients, both the initial and the metachronous lesions occurred in the distal two-thirds portion of the stomach. Except for two lesions that were 30 and $40 \mathrm{~mm}$ in size, the median size of the MGCs was $10.3 \pm 5.4 \mathrm{~mm}$ (range, $2-20 \mathrm{~mm}$ ), compared to $14.9 \pm 7.8 \mathrm{~mm}$ (range, $5-30 \mathrm{~mm}$ ) for the initial lesions. The histology of the MGCs consisted of well- or moderately differentiated adenocarcinomas.
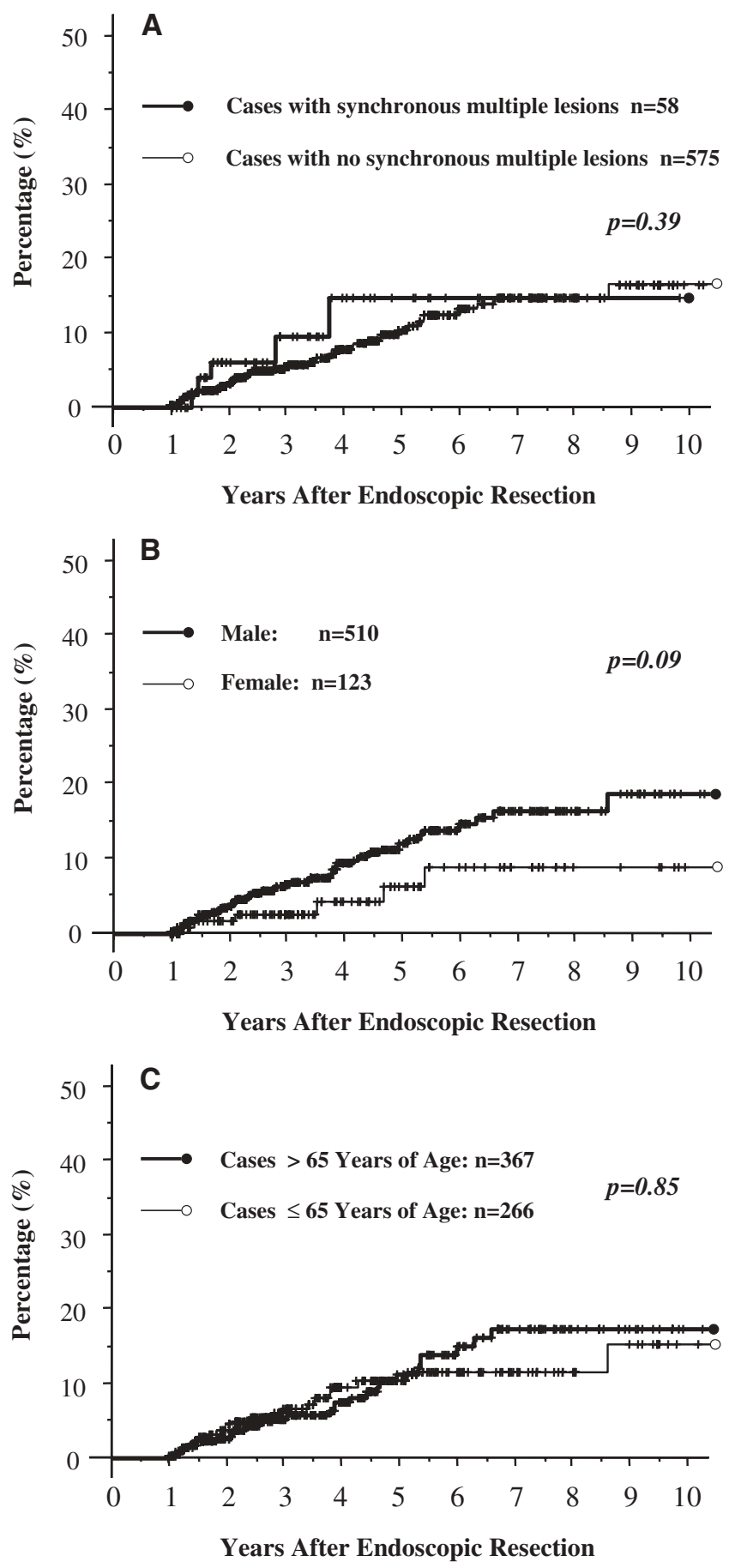

Fig. 3. A Cumulative incidence of MGC in patients who had synchronous lesions (lesions found within 1 year of their initial treatment) and in patients who had no synchronous lesions. B Cumulative incidence of MGC by sex. C Cumulative incidence of MGC by age. None of the three factors shown in $\mathbf{A}$, $\mathbf{B}$, and $\mathbf{C}$ had a statistically significant effect on the incidence of MGC

\section{Outcomes}

All first MGCs met the indications for ER and were treated by repeat ER within 2 months of detection, with 
Table 3. Patients with noncuratively resected metachronous lesions

\begin{tabular}{|c|c|c|}
\hline & Patient 1 & Patient 2 \\
\hline Age at onset of metachronous lesion (years) & 71 & 76 \\
\hline Lesion size $(\mathrm{mm})$ & 20 & 10 \\
\hline Sex & Male & Male \\
\hline $\begin{array}{l}\text { Interval between initial treatment and detection of } \\
\text { metachronous lesion (years) }\end{array}$ & 5.3 & 3.9 \\
\hline Macroscopic type & IIc & IIc \\
\hline Location & Upper third & Lower third \\
\hline \multicolumn{3}{|l|}{ Pathological results } \\
\hline Differentiation & Well & Moderate \\
\hline Undifferentiated component ${ }^{\mathrm{a}}$ & $\mathrm{Yes}^{\mathrm{b}}$ & Yes $^{\mathrm{b}}$ \\
\hline Lymphovascular Invasion & No & No \\
\hline Depth of invasion & $500 \mu \mathrm{m}$ & Intramucosal \\
\hline Additional treatment & None & None \\
\hline $\begin{array}{l}\text { Interval between latest endoscopic examination and } \\
\text { detection of metachronous lesion (years) }\end{array}$ & 3 & 0.5 \\
\hline
\end{tabular}

pathologically curative resections being obtained in 50 patients $(96.2 \%)$; the repeat ER was judged to be noncurative in 2 patients (Table 3 ). With respect to the subjects of this study, there have been no tumor-related deaths, and the incidence of noncurative MGC was only $0.3 \%$ ( 2 out of 633 patients), with both of these patients choosing further observation rather than additional treatment.

\section{The two patients with noncurative $M G C$ resections}

Patient 1 was a 71-year-old man. The interval between the latest endoscopic examination and discovery of the MGC was 3 years. The depth of invasion of the metachronous lesion was classified as superficial submucosa $(500 \mu \mathrm{m})$. There was no lymphovascular involvement, but the lesion contained a poorly differentiated component in the deepest portion. The patient refused additional surgery, however, and chose continued observation instead.

Patient 2 was a 76-year-old man, and the metachronous lesion was classified endoscopically as a small 0-IIc lesion. The histopathological finding of the resected specimen revealed intramucosal cancer and moderate to poorly differentiated adenocarcinoma, but the patient refused additional surgery because of his advanced age. The interval between the latest endoscopic examination and discovery of the MGC was 6 months.

\section{Follow-up interval}

Regarding the intervals between follow-up examinations, we focused on the 52 patients with metachronous lesions. The median time interval from the last exami- nation to the actual discovery of a metachronous lesion was $1.0 \pm 0.6$ years (range, $0.2-3.1$ years).

The percentage of patients whose interval from the last examination to the discovery of a metachronous lesion was less than 1 year was $46.2 \%$ (24/52). Of these 24 patients, 13 had received earlier follow-up examinations due to the presence of adenomas or suspicion of carcinomas, because such patients were followed more intensively. If such a lesion was definitely evaluated as being a well-differentiated adenocarcinoma, it was resected.

Earlier follow-up examinations had been performed for five other patients at their request. As for the remaining six patients, previous examinations had been performed for limited purposes, such as the checking of gastric ulcer healing and esophagus screening, but these examinations were considered to be insufficient for detecting a metachronous lesion, and when taken into account, reduced the average time interval.

\section{Discussion}

We have shown here the overall incidence and the annual incidence of MGC. In regard to the post-ER surveillance program at our institution, annual endoscopic examinations were performed for patients who had undergone curative ER. In this study, there were no patients with tumor-related death due to a MGC. For one of the two patients with noncurative metachronous lesion resection (patient 2), the interval between the last examination and discovery of the MGC was 6 months, and the depth of invasion was intramucosal. The percentage of these patients with noncurative resections 
was very small, at $0.3 \%$ ( 2 out of 633 patients). In addition, the average time interval from the last examination to the actual discovery of a metachronous lesion was approximately 1 year. Although we could adopt a more extensive endoscopic surveillance program, undifferentiated-type gastric cancer might still occur within the surveillance period and we could not prevent any such undifferentiated type MGC. Our annual endoscopic surveillance program for the early detection of metachronous lesions was considered to be both practical and effective. The ideal approach for evaluating a screening program following ER, of course, would be to observe patients prospectively.

As for predictive factors, synchronous multiplicity of the gastric cancer and the patient's age at the time of the initial ER have been reported to significantly affect the incidence of metachronous lesions [20], but these factors did not significantly affect this study's results.

Recent progress in endoscopic examination has made it possible to detect small EGCs, and ER for EGCs has been widely accepted as a less invasive treatment. Of note, a new one-piece resection method, known as ESD, which uses several special endoscopic tools, including an insulation-tipped diathermic knife [14], was developed with a standard single-channel gastroscope. Compared with conventional EMR procedures, ESD has several advantages: (1) larger lesions can be resected and completely removed in one piece; (2) lesions with ulcer scars can be resected; (3) it is much easier to ensure adequate resection margins by directly visualizing the actual cut line around the lesion; and (4) it is possible to resect lesions located on the lesser curvature and posterior wall, which are considered to be more difficult to resect by EMR. As a result, such onepiece resections allow for an expansion of the indications for ER.

There have been reports of cancer of the gastric remnant occurring in $1.8 \%$ to $5 \%$ of patients who have had surgical treatment for gastric cancer [11,21], so the remnant stomach after such treatment is at high risk for the development of MGC. Because ER can preserve more of the stomach than surgical resection, MGC recurrence at other gastric sites after ER has become a major concern, and its early detection is critical.

Frequent follow-up examinations limit a patient's quality of life, however, and contribute to an increase in overall medical expenses. For the present, annual endoscopic examinations are considered to be acceptable. In the future, appropriate surveillance systems, which include the evaluation of several risk factors for gastric carcinogenesis, such as the existence of microorganisms, a patient's dietary habits, and genetic and epigenetic events, should be investigated and established.

For instance, a recent study reported that the eradication of Helicobacter pylori after ER of EGC decreased the incidence of MGC [22]. In the field of genetic and epigenetic events, mutations of the E-cadherin and $p 53$ genes are reported to be involved in the multicentric carcinogenesis of diffuse gastric cancer, especially hereditary diffuse gastric cancer [23,24]. It was also reported that patients with microsatellite instability (MSI)-positive gastric cancers had a significantly higher risk of secondary cancer than patients with MSInegative cancers [25]. DNA methylation is one of the epigenetic changes which have recently emerged as important causes of carcinogenesis. In noncancerous lesions, aberrant $\mathrm{CpG}$ island methylation of several genes has also been reported [26-29]. In an effort to distinguish patients at high risk for metachronous lesions from those at low risk, clarification of molecular alterations that are closely linked with such characteristics would be beneficial, especially after ER.

\section{References}

1. Gotoda T, Yanagisawa A, Sasako M, Ono H, Nakanishi Y, Shimoda $\mathrm{T}$, et al. Incidence of lymph node metastasis from early gastric cancer: estimation with a large number of cases at two large centers. Gastric Cancer 2000;3:219-25.

2. Yamao T, Shirao K, Ono H, Kondo H, Saito D, Yamaguchi H, et al. Risk factors for lymph node metastasis from intramucosal gastric carcinoma. Cancer 1996;77:602-6.

3. Yasuda K, Shiraishi N, Suematsu T, Yamaguchi K, Adachi Y, Kitano S. Rate of detection of lymph node metastasis is correlated with the depth of submucosal invasion in early stage gastric carcinoma. Cancer 1999;85:2119-23.

4. Moertel CG, Bargen JA, Soule EH. Multiple gastric cancers; review of the literature and study of 42 cases. Gastroenterology 1957:32:1095-103.

5. Kosaka T, Miwa K, Yonemura Y, Urade M, Ishida T, Takegawa $\mathrm{S}$, et al. A clinicopathologic study on multiple gastric cancers with special reference to distal gastrectomy. Cancer 1990;65:2602-5.

6. Esaki Y, Hirokawa K, Yamashiro M. Multiple gastric cancers in the aged with special reference to intramucosal cancers. Cancer 1987;59:560-5.

7. Kodera Y, Yamamura Y, Torii A, Uesaka K, Hirai T, Yasui K, et al. Incidence, diagnosis and significance of multiple gastric cancer. Br J Surg 1995;82:1540-3.

8. Iwafuchi M, Watanabe H. Synchronous multiple early gastric cancer; study of surgically resected stomach (in Japanese). Nippon Rinsho (Jpn J Clin Med) 1996;54:1217-23.

9. Mitsudomi T, Watanabe A, Matsusaka T, Fujinaga Y, Fuchigami T, Iwashita A. A clinicopathological study of synchronous multiple gastric cancer. Br J Surg 1989;76:237-40.

10. Honmyo U, Misumi A, Murakami A, Haga Y, Akagi M. Clinicopathological analysis of synchronous multiple gastric carcinoma. Eur J Surg Oncol 1989;15:316-21.

11. Takeda J, Toyonaga A, Koufuji K, Kodama I, Aoyagi K, Yano S, et al. Early gastric cancer in the remnant stomach. Hepatogastroenterology 1998;45:1907-11.

12. Hosokawa O, Kaizaki Y, Watanabe K, Hattori M, Douden K, Hayashi $\mathrm{H}$, et al. Endoscopic surveillance for gastric remnant cancer after early cancer surgery. Endoscopy 2002;34:469-73.

13. Tada M, Murakami A, Karita M, Yanai H, Okita K. Endoscopic resection of early gastric cancer. Endoscopy 1993;25:445-50.

14. Ono H, Kondo H, Gotoda T, Shirao K, Yamaguchi H, Saito D, et al. Endoscopic mucosal resection for treatment of early gastric cancer. Gut 2001;48:225-9. 
15. Eguchi T, Gotoda T, Ichiro O, Hamanaka H, Noriaki H, Saito D. Is endoscopic one-piece mucosal resection essential for early gastric cancer? Dig Endosc 2003;15:113-6.

16. Oda I, Gotoda T, Hamanaka H. Endoscopic submucosal dissection for early gastric cancer: technical feasibility, operation time and complications from a large consecutive series. Dig Endosc 2005;17:54-8

17. Japanese Gastric Cancer Association. Japanese classification of gastric carcinoma, 2nd English edition. Gastric Cancer 1998;1:10 24.

18. Kajitani T. The general rules for the gastric cancer study in surgery and pathology. Part I. Clinical classification. Jpn J Surg 1981;11:127-39.

19. The general rules for the gastric cancer study in surgery and pathology. Part II. Histological classification of gastric cancer. Jpn J Surg 1981;11:140-5.

20. Arima N, Adachi K, Katsube T, Amano K, Ishihara S, Watanabe $\mathrm{M}$, et al. Predictive factors for metachronous recurrence of early gastric cancer after endoscopic treatment. J Clin Gastroenterol 1999:29:44-7.

21. Nicholls JC. Stump cancer following gastric surgery. World J Surg 1979;3:731-6.

22. Uemura N, Okamoto S. Effect of Helicobacter pylori eradication on subsequent development of cancer after endoscopic resection of early gastric cancer in Japan. Gastroenterol Clin North Am 2000;29:819-27.
23. Fitzgerald RC, Caldas C. Clinical implications of E-cadherin associated hereditary diffuse gastric cancer. Gut 2004;53:7758.

24. Charlton A, Blair V, Shaw D, Parry S, Guilford P, Martin IG. Hereditary diffuse gastric cancer: predominance of multiple foci of signet ring cell carcinoma in distal stomach and transitional zone. Gut 2004;53:814-20.

25. Miyoshi E, Haruma K, Hiyama T, Tanaka S, Yoshihara M, Shimamoto F, et al. Microsatellite instability is a genetic marker for the development of multiple gastric cancers. Int J Cancer 2001; 95:350-3.

26. Waki T, Tamura G, Tsuchiya T, Sato K, Nishizuka S, Motoyama T. Promoter methylation status of E-cadherin, hMLH1, and p16 genes in nonneoplastic gastric epithelia. Am J Pathol 2002;161: 399-403.

27. Waki T, Tamura G, Sato M, Motoyama T. Age-related methylation of tumor suppressor and tumor-related genes: an analysis of autopsy samples. Oncogene 2003;22:4128-33.

28. To KF, Leung WK, Lee TL, Yu J, Tong JH, Chan MW, et al. Promoter hypersmethylation of tumor-related genes in gastric intestinal metaplasia of patients with and without gastric cancer. Int J Cancer 2002;102:623-8.

29. Honda T, Tamura G, Waki T, Kawata S, Nishizuka S, Motoyama T. Promoter hypermethylation of the Chfr gene in neoplastic and non-neoplastic gastric epithelia. Br J Cancer 2004;90:20136. 